\title{
Effective Electromagnetic Properties of Woven Fiber Composites for Shielding Applications
}

\author{
Ghida Al Achkar, Lionel Pichon, Laurent Daniel and Nabil Benjelloun
}

\begin{abstract}
Composite materials reinforced with woven conductive fibers can be good candidates for electromagnetic shielding applications. Their light weight provides an important advantage over metallic alloys classically used in the automotive and aircraft industries. However, numerical modeling of composite-based large-scale structures such as shielding enclosures, is rendered almost impossible by the heterogeneities at the microscopic scale. This problem is commonly addressed using electromagnetic homogenization methods. In this paper, we propose a homogenization technique based on finite element computations and inverse problem solving for estimating the effective properties of woven composites. The effect of electrical contacts between the fibers is also studied and its influence on the global behavior of the material is analyzed. The results are then shown to diverge from those obtained using analytical mixing rules over the frequency range of 1 to $40 \mathrm{GHz}$. Thus the suitability of these homogenization methods is discussed with respect to the studied frequency range and the nature of the microstructure.
\end{abstract}

Index Terms-Effective media, composite materials, 3D finite element method, homogenization, optimization, shielding effectiveness, electrical contact.

\section{INTRODUCTION}

$\mathbf{T}$ HE increased use of composite materials in the automotive and aircraft industries is mainly due to their mechanical properties and reduced weight. However, the electrical properties of certain types of composites can make them good candidates for replacing metal alloys in electromagnetic compatibility applications. Studying the electromagnetic (EM) response of composite materials in the vicinity of electronic devices becomes necessary, especially for incorporating such materials in the design of large-scale structures such as shielding enclosures. Modeling these structures with typical numerical methods (finite element method FEM, finite difference time domain FDTD) is nonetheless computationally heavy even when the enclosure is made of homogeneous conductive materials (i.e. aluminum alloys). Implementing composite-based structure models using numerical methods becomes impossible because of the heterogeneous nature of composite materials at the microscopic scale. It is useful to use homogenization methods as solutions for this computational problem. Once a homogeneous equivalent medium for the composite is defined through its complex permittivity and

G. Al Achkar, L. Pichon and L. Daniel are with the Laboratoire de Génie Électrique et Électronique de Paris GeePs, CNRS (UMR 8507) / CentraleSupélec / UPMC / Univ. Paris-Sud / Univ. Paris-Saclay, 91192 Gif-sur-Yvette, France (email: ghida.al-achkar@ centralesupelec.fr; lionel.pichon@centralesupelec.fr; laurent.daniel@centralesupelec.fr).

N. Benjelloun is with the Institut de Recherche en Systèmes Électroniques Embarqués, IRSEEM-ESIGELEC-EA 4353, 76801 Saint-Etienne du Rouvray, France (email: nabil.benjelloun@esigelec.fr). permeability the problem is reduced to solving a standard case of shielding structure.

This idea constitutes a good starting point for the study of woven fiber composites, a material that is widely used, and manufactured through well known processes. The complexity of the geometry as well as the anisotropy of the material make it necessary to define a proper homogenization technique. To this end, a study of the literature shows that numerous EM homogenization methods can be used. They are grouped under three main categories: analytical models, numerical methods and experimental setups.

Analytical mixing rules [1] can either provide lower and upper bounds of the effective properties, such as Wiener (W) and Hashin-Shtrikman bounds [2], or estimates of the EM properties under certain assumptions regarding the material and excitation (Maxwell-Garnett, Bruggmann, ...). Their application domain can vary according to a multitude of parameters, including but not limited to frequency, filling factor, and polarization of the EM wave with respect to the heterogeneities or the number of constituents. These formulas have been used to estimate the effective EM properties of various composites over different frequency ranges [3]-[7]. While these analytical mixing rules are fast and efficient, their suitability must each time be verified numerically or experimentally.

As for numerical methods, they are most helpful at the design stage as they allow for parametric examinations of different constituent properties and dimensions. They can however be computationally heavy especially for three dimensional models. Different numerical methods can be used to study the EM behavior of composite materials. At the microscopic scale, the homogenization problem relies on the FEM to extract the average electrical properties [8]-[10]. At the mesoscopic scale, which is that of the woven fabric, numerical methods are used either to compute the effective properties directly, or through EM parameters that are closely related to them, such as the transmission and reflection coefficients. More concretely, computational methods like rigorous coupled wave analysis [11] and full wave expansion [12] are not well suited for electrically conductive materials. On the other hand, methods like FDTD [13], [14] can be used to model woven carbon fiber reinforced polymers by assuming a perfect conductor medium, or also to model thin composite panels. Additionally, other numerical techniques [15], [16] like circuital approaches and transfer matrix method can be used to compute the effective properties of woven fabrics and metallic wire grids over different frequency ranges.

In this paper, the 3D problem of modeling woven fiber composites is studied using the FEM. The complexity of the 
geometry, as well as the skin effect to be considered in the gigahertz band makes this method a suitable one. As for the extraction of the effective properties, experimental techniques [17], [18] have been combined with appropriate inverse problem solving to provide a good estimate of the effective properties of conductive fiber composite materials. However, in this paper a simulation-based homogenization technique is proposed. It allows for a practical study of the contribution of different parameters (fiber contact, conductivity) to the woven material's behavior. Additionally, it is helpful for studying non symmetric composites as the polarization of the incident wave with respect to the inclusion can be predefined. In the first part, this technique based on finite element computations and inverse problem solving is presented. In a previous work [19], a simple technique was introduced. Based only on the shielding coefficient, it does not effectively capture the material behavior because it does not take into consideration the reflection part of its response. Moreover it was applied to a 2D model of a unidirectional fiber composite. Hereinafter, this method is extended for a comprehensive description of the material. It is also applied to a $3 \mathrm{D}$ model of a woven fabric composite that takes into account electrical contacts between the fibers. In the second part, a simplified model of the material is presented in an attempt to explore the applicability of analytical formulas for the structure at hand. In the last part the obtained results are analyzed and a conclusion is drawn.

\section{Proposed homogenization METHod GENERAL OUTLINE}

The estimation of materials EM properties involves the evaluation of an intermediate parameter from which these properties can be extracted. In our case, the shielding coefficient [20] (Shielding Effectiveness $S E_{d B}$ ) and the reflection coefficient $R_{d B}$ will be used to extract the effective electrical properties. This method relies on the following two main steps (Fig. 1) :

- (Step 1) Computing $S E_{d B}$ and $R_{d B}$ using a finite element method: by carrying out the simulations detailed in section III. the plane-wave shielding and reflection coefficients of an infinite composite sheet can be evaluated over a broadband of frequencies.

- (Step 2) Solving an inverse problem to obtain the effective properties: once the simulated coefficients for the heterogeneous sheet are obtained from Step 1, a definition of EM homogenization is then used to extract the composite effective complex permittivity. The principle of homogenization being to replace a heterogeneous plate with its homogeneous equivalent, the logic behind the chosen technique is as follows: for each frequency of the incident wave, there exists a pair of effective permittivity and conductivity $\left(\epsilon=\epsilon_{e f f} ; \sigma=\sigma_{e f f}\right)$ associated with a fictitious homogeneous electrical medium that will give the same shielding and reflection coefficients as those obtained for the composite material simulated numerically. Moreover, $S E_{d B}$ and $R_{d B}$ are a function of these effective properties $\left(\epsilon_{e f f} ; \sigma_{e f f}\right)$ and other known parameters as shown in (2)-(6). As a result, finding the effective properties means minimizing the cost function $F_{c}$.

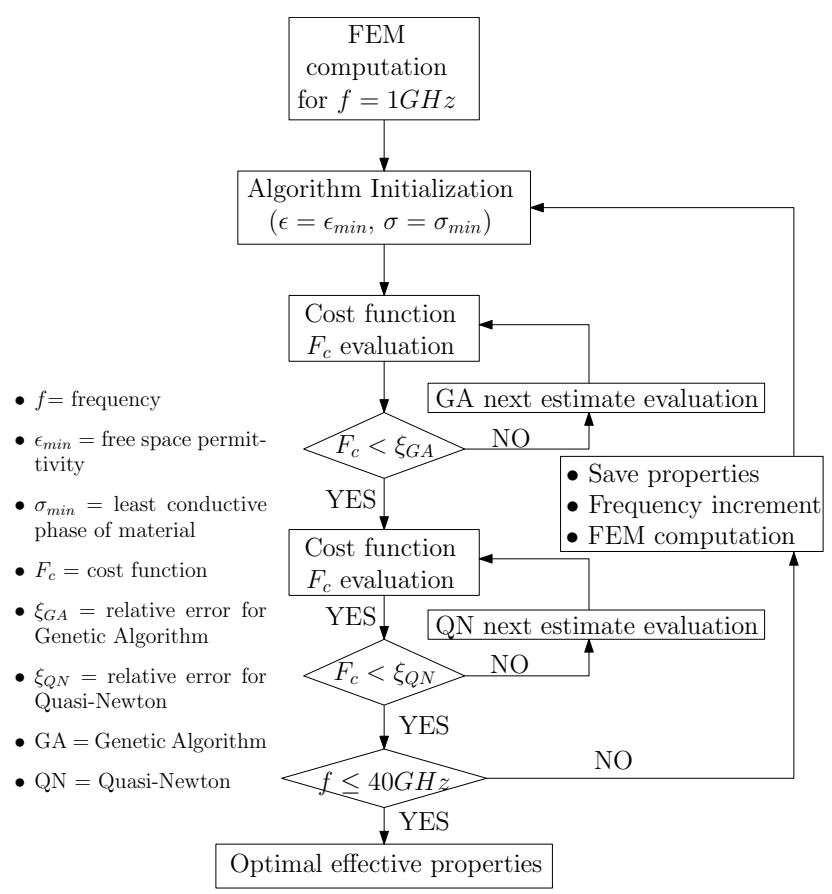

Fig. 1: General scheme of the proposed homogenization technique.

$$
\begin{aligned}
F_{c} & =a\left|S E_{F E M}(f)-S E_{\text {analytical }}(\epsilon(f), \sigma(f))\right|_{d B}^{2} \\
& +b\left|R_{F E M}(f)-R_{\text {analytical }}(\epsilon(f), \sigma(f))\right|_{d B}^{2}
\end{aligned}
$$

where $S E_{F E M}, R_{F E M}, S E_{\text {analytical }}, R_{\text {analytical }}$ are the FEM and analytical estimates of the shielding and reflection coefficients. $\epsilon$ is the electrical permittivity, $\sigma$ is the electrical conductivity and $f$ is the frequency of the incident EM wave. The cost function is written in terms of both the reflection coefficient and the shielding effectiveness. Indeed, in [19], the optimization problem is formulated in terms of only $S E$. However, an in depth examination of the previous results showed that they did not adequately fit the reflection coefficient. Moreover, in order to get the best results out of the minimization study, two weighing coefficients $a$ and $b$ are added. This type of regularization shows to give a good estimate of the minimum for the values: $a(f)=\left(1,\left|R_{F E M}(f)\right|\right)$, $b(f)=\left(1,\left|S E_{F E M}(f)\right|\right)$. Choosing $a$ and $b$ 's values remains to be determined according to the studied material.

Expressed in $\mathrm{dB}$, the shielding effectiveness $S E_{d B}=$ $S E_{\text {analytical }}$ of a homogeneous infinite sheet subjected to a normally incident plane wave groups three main phenomena: the absorption of the material $S E_{A}$, the reflection part $S E_{R}$ and the multiple reflections that occur inside the plate $S E_{B}$ [21].

$$
\begin{gathered}
S E_{d B}=S E_{A}+S E_{B}-S E_{R} \quad \text { where }: \\
\left\{\begin{array}{l}
S E_{A}=20 \log _{10}\left(\left|e^{\jmath k l}\right|\right) \\
S E_{B}=20 \log _{10}\left(\left|1-q e^{\jmath 2 k l}\right|\right) \\
S E_{R}=20 \log _{10}(|g|)
\end{array}\right.
\end{gathered}
$$


with $k=\sqrt{\epsilon \mu \omega^{2}-\jmath \mu \sigma \omega}$ the wave vector inside the plate. $(\epsilon, \sigma)$ are the permittivity and conductivity of the plate. $l$ is the plate thickness and $\omega$ the angular frequency of the propagating wave. The parameters $g$ and $q$ are functions of the electromagnetic properties of the plate:

$$
\left\{\begin{array}{l}
g=\frac{4 \eta \eta_{0} \mu_{r}}{\left(\eta_{0}+\eta \mu_{r}\right)} \\
q=\left(\frac{\eta_{0}-\eta \mu_{r}}{\eta_{0}+\eta \mu_{r}}\right)^{2}
\end{array}\right.
$$

with $\eta=\frac{\mu_{0} \omega}{k}$ the impedance of the plate and $\mu_{r}$ the relative permeability. The reflection coefficient $R_{d B}$ is defined as the ratio of the reflected wave to the incident wave:

$$
R_{d B}=20 \log _{10} \frac{\left|\boldsymbol{E}_{\boldsymbol{R}}\right|}{\left|\boldsymbol{E}_{\boldsymbol{I}}\right|}
$$

which when developed gives:

$$
R_{d B}=20 \log _{10} \frac{\left(\eta_{0}-\eta\right)\left(e^{j 2 k l}-1\right)}{\left(\eta_{0}+\eta\right)\left(q-e^{\jmath 2 k l}\right)}
$$

These equations are used in the minimization process in order to extract the effective properties of the composite. As for the optimization algorithm, to ensure a fast convergence, a combination of a genetic [22] and a deterministic algorithm is used. Genetic algorithms are well known for their repeated modification of the population of individual solutions thus avoiding local minima. This algorithm is used to roughly localize the global minimum within a certain error margin, a deterministic quasi-Newton algorithm is then carried out from the stopping point of the previous algorithm for faster convergence towards the global minimum of the cost function.

In the next part, the behavior of woven fiber composites is studied through finite element computations. The obtained shielding and reflection coefficients are used to compute the effective properties with respect to frequency.

\section{ELECTROMAGNETIC BEHAVIOR AND HOMOGENIZATION OF WOVEN FIBER COMPOSITES}

\section{A. Finite element computations}

Shielding properties of materials are estimated through the ratio of the incident wave $\left|\boldsymbol{E}_{\boldsymbol{I}}\right|$ to the transmitted wave $\left|\boldsymbol{E}_{\boldsymbol{T}}\right|$, called shielding effectiveness $(S E)$, and defined as follows:

$$
S E_{d B}=20 \log _{10} \frac{\left|\boldsymbol{E}_{\boldsymbol{I}}\right|}{\left|\boldsymbol{E}_{\boldsymbol{T}}\right|}
$$

A periodic cell of the studied composite is shown in Fig. 2, it contains a plain weave fabric made of conductive fibers drowned in a layer of dielectric material. In order to compute the shielding effectiveness of this geometrically complex material, a finite element approach is adopted using the commercial software COMSOL ${ }^{\mathrm{TM}}$. The computation domain (Fig. 3) contains a 3-D unit cell of the material surrounded by two layers of air. Two perfectly matched layers (PML) are added to prevent undesirable reflections by simulating an infinite medium around the domain [23]. The sheet is illuminated by a plane wave $\left(\boldsymbol{E}_{\boldsymbol{I}}\right)$ polarized in the $\boldsymbol{u}_{\boldsymbol{x}}$ direction.

$$
\boldsymbol{E}_{\boldsymbol{I}}(z, t)=E_{I} e^{\left(\jmath \omega t-k_{0} z\right)} \boldsymbol{u}_{\boldsymbol{x}}
$$

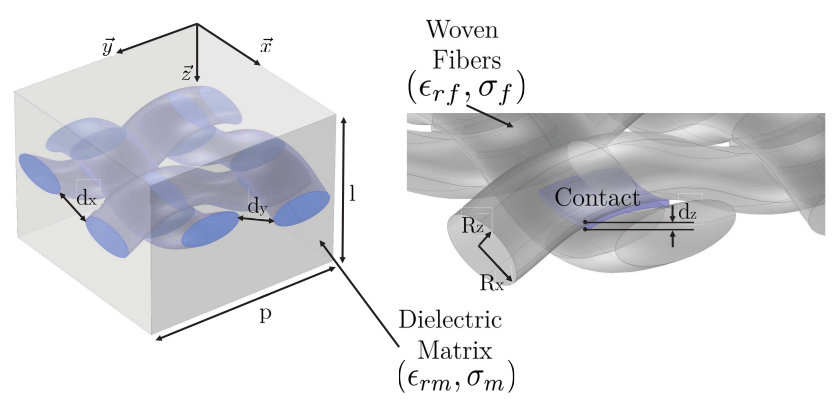

Fig. 2: Woven fiber composite.

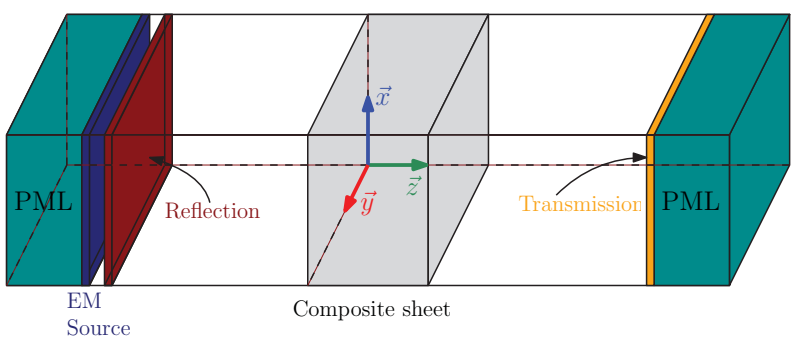

Fig. 3: 3D - Finite element computation domain.

with $k_{0}=\frac{\omega}{c}$ the free space wavenumber $\left(c=3.10^{8}\right.$ m.s $\left.s^{-1}\right)$ and $\omega$ the angular frequency of the propagating wave.

Frequency domain simulations provide an estimate of shielding effectiveness. At first, the validity of the FEM model is verified by matching the simulated shielding effectiveness of a homogeneous sheet to that obtained by applying the analytical formula detailed in (2)-(6). A sample of the material is then studied, the results represented in Fig. 4 show a pattern of behavior for different values of fiber conductivity $\sigma_{f}$. The non-magnetic composite essentially acts as a highpass filter ensuring acceptable levels of attenuation in the lower frequency range.

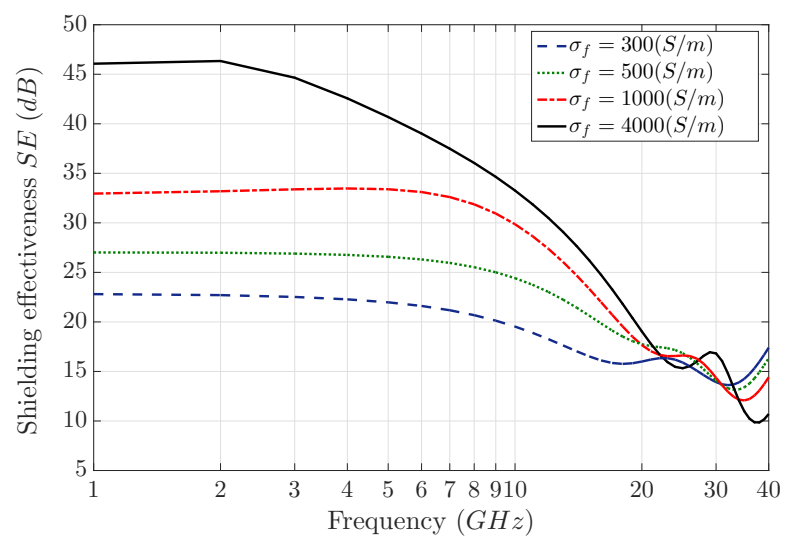

Fig. 4: Shielding effectiveness of an infinite sheet (woven fiber microstructure of Fig. 2) for various fiber conductivities $\sigma_{f}$. Configuration: electric field polarized in the $\boldsymbol{x}$ direction, fiber concentration ratio $f_{v}=17.5 \%, l=3 \mathrm{~mm}, p=4 \mathrm{~mm}, d_{x}=$ $d_{y}=1.22 \mathrm{~mm}, d_{z}=140 \mu \mathrm{m}, R_{x}=625 \mu \mathrm{m}, R_{z}=250 \mu \mathrm{m}$, $\epsilon_{r f}=1,\left(\epsilon_{r m}, \sigma_{m}\right)=(5,0 \mathrm{~S} / \mathrm{m})$. 
In [16], shielding effectiveness for different metallic wire meshes is measured. In order to validate the finite element model presented here, a simulation of the woven fabric (sample S1 in [16]) with the following dimensions and properties has been conducted: $l=270 \mu \mathrm{m}, p=1.116 \mathrm{~mm}, d_{x}=d_{y}=$ $508 \mu \mathrm{m}, d_{z}=15 \mu \mathrm{m}, R_{x}=25 \mu \mathrm{m}, R_{z}=25 \mu \mathrm{m},\left(\epsilon_{r f}, \sigma_{f}\right)=$ $\left(1,5.8 \cdot 10^{7} \mathrm{~S} / \mathrm{m}\right),\left(\epsilon_{r m}, \sigma_{m}\right)=\left(3.4,50 \cdot 10^{-9} \mathrm{~S} / \mathrm{m}\right)$. It should be noted that the finite element model studied here is suitable to simulate this geometry. Fig. 5 shows the obtained results. Over the frequency range of $1 \mathrm{GHz}$ to $100 \mathrm{GHz}$, the obtained results match those of conducted study, with a few differences that can be caused by quality of the mesh when approaching the higher frequencies.

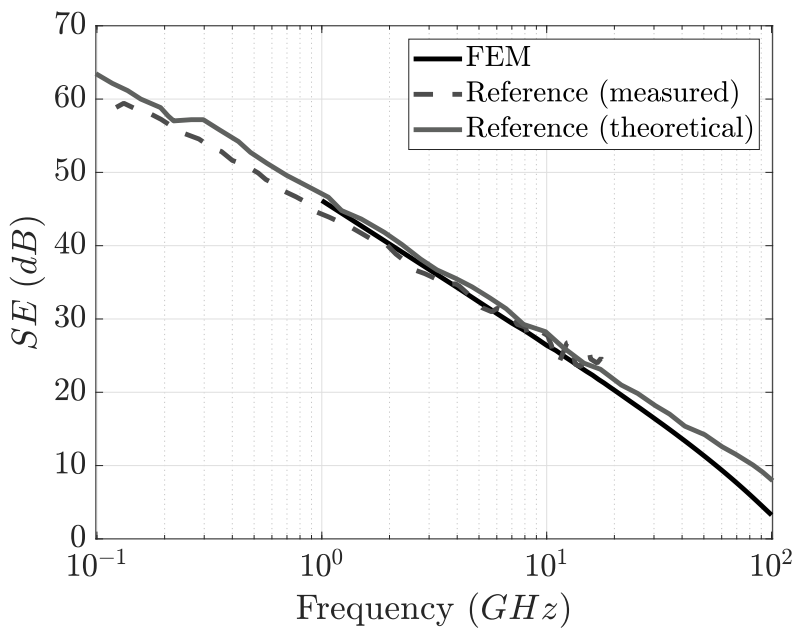

Fig. 5: Shielding effectiveness of an infinite sheet (woven fiber microstructure of Fig. 2) compared to simulated and measured data obtained from [16] (sample S1). Configuration: electric field polarized in the $\boldsymbol{x}$ direction, $l=270 \mu \mathrm{m}, p=1.116 \mathrm{~mm}$, $d_{x}=d_{y}=508 \mu m, d_{z}=15 \mu m, R_{x}=25 \mu \mathrm{m}, R_{z}=25 \mu \mathrm{m}$, $\left(\epsilon_{r f}, \sigma_{f}\right)=\left(1,5.8 \cdot 10^{7} \mathrm{~S} / \mathrm{m}\right),\left(\epsilon_{r m}, \sigma_{m}\right)=\left(3.4,50 \cdot 10^{-9} \mathrm{~S} / \mathrm{m}\right)$.

The manufacturing process of woven composite materials establishes an elastic contact between the fibers [24]. This results in a variation of the electrical resistivity of the contact area according to [25]. This effect is integrated into the FEM model by adding thin layers of conductive materials between the fibers to represent the electric contact. By varying the surface of these layers the effect of the contact ratio can be considered. Fig. 6 shows that vertical contact between fibers reinforces shielding effectiveness by introducing closed paths for the induced currents to circulate. Because the contact ratio between fibers is dependent on the weaving and resin molding process (as well as other mechanical and thermal influencing factors), it varies from one weave to another inside the same fabric. Its estimation should therefore be based on experimental data. Moreover, the study of contacts can be more complicated due to asperities [26]. In this case the contact area can indeed be obtained by numerical fitting.

\section{B. Homogenization results}

The proposed homogenization technique is carried out for the woven fiber composite of Fig. 2. The computed effective

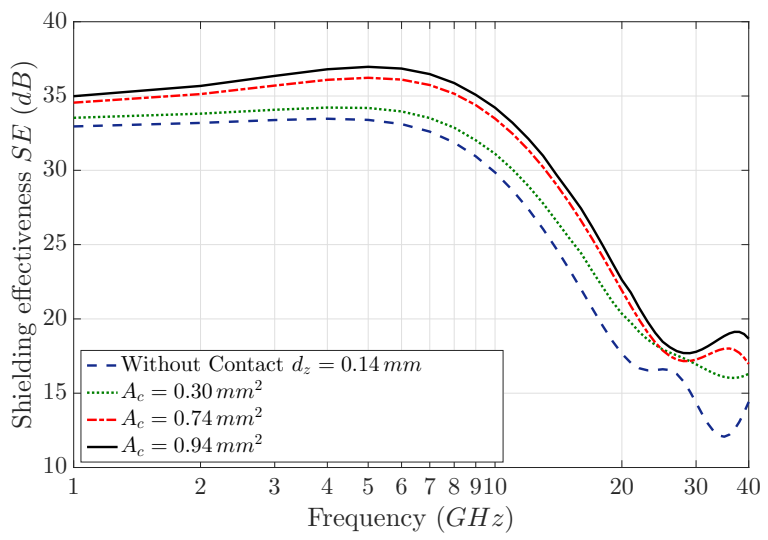

Fig. 6: Shielding effectiveness of an infinite sheet (woven fiber composites of Fig. 2): variation according to contact surface area $A_{c}$. Configuration: electric field polarized in the $\boldsymbol{x}$ direction, fiber concentration ratio $f_{v}=17.5 \%, l=3 \mathrm{~mm}$, $p=4 \mathrm{~mm}, d_{x}=d_{y}=1.22 \mathrm{~mm}, R_{x}=625 \mu \mathrm{m}, R_{z}=$ $250 \mu m,\left(\epsilon_{r f}, \sigma_{f}\right)=\left(1,10^{3} \mathrm{~S} / \mathrm{m}\right),\left(\epsilon_{r m}, \sigma_{m}\right)=(5,0 \mathrm{~S} / \mathrm{m})$.

properties as well as the shielding effectiveness and reflection coefficient estimates computed using this technique are plotted in Fig. 7 (in this case study, the weighing coefficients are: $a=\left|R_{F E M}(f)\right|$ and $\left.b=\left|S E_{F E M}(f)\right|\right)$. Results demonstrate a good agreement between numerical calculations and the homogenized medium, with an estimated error of approximately $5 \%$ in this case. Additionally, multiple studies consisting in carrying out this homogenization method over different woven fiber composites (parametric studies) demonstrate its tendency to capture with good accuracy their frequency behavior by computing a corresponding effective medium (Fig. 8). Here the used weighing coefficients are: $a=\left|R_{F E M}(f)\right|$ and $b=1$. The obtained results show that by simulating a unit cell of a heterogeneous material, we are able to define an equivalent homogeneous medium at the macroscopic scale. This is useful when conducting large-scale simulations of realistic structures for electromagnetic compatibility applications.

\section{EQUiVAlENT TWO LAYER MODEL}

In order to evaluate the woven fiber model presented in the previous section, a comparison with analytical formulas was performed to check their ability to predict the frequency response. To this end a simplified model of the woven composite is proposed as a stepping stone to applying analytical mixing rules. The similarities between the two materials are detailed at first, the simulation results are then analyzed.

From an electrical viewpoint, the woven fiber microstructure is essentially made of two sets of fibers. A set of unidirectional fibers laying perpendicularly to the incident electric field, and a second set of the same fibers which are parallel to the electric field. Moreover, the material being diagonally anisotropic, the effective permittivity takes the form of a diagonal dyadic. Additionally, the symmetry in the $(x y)$ plane gives $\epsilon_{x}^{*}=\epsilon_{y}^{*}$. This is also the case for a composite material containing two layers of unidirectional fibers set in opposite directions (see Fig. 9). 

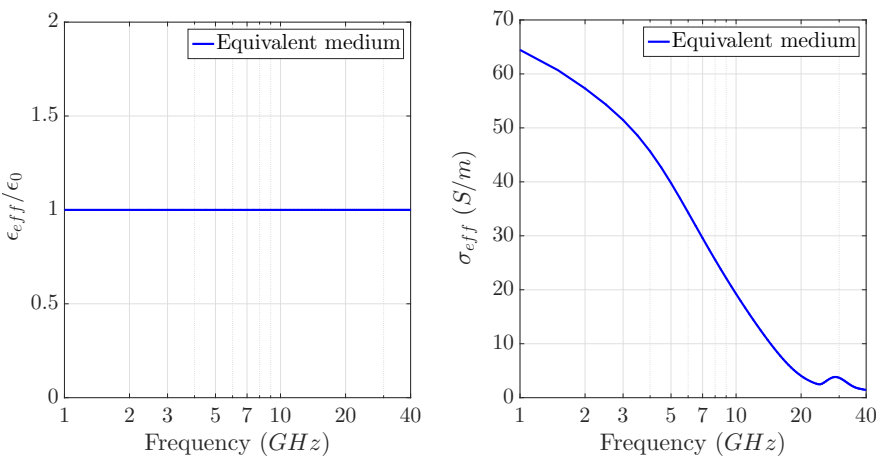

(a) Effective properties (permittivity and conductivity).
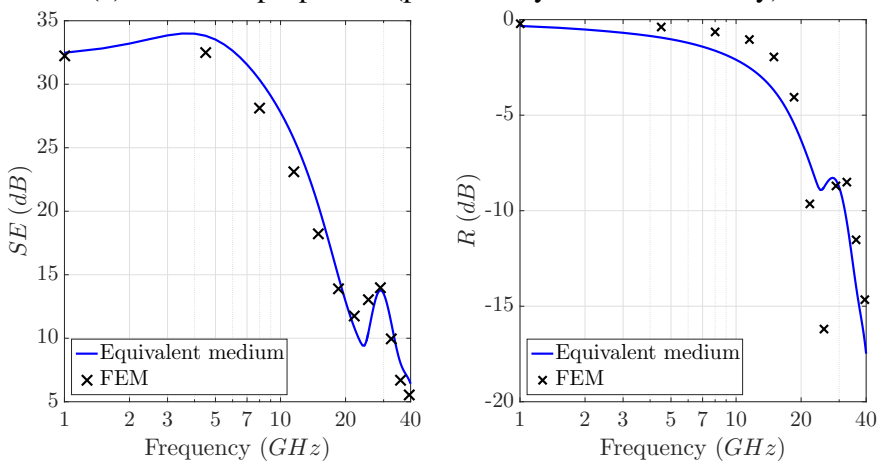

(b) Shielding and reflection coefficients.

Fig. 7: FEM and homogenization results of a woven fiber composite. Configuration: electric field polarized in the $x$ direction, fiber concentration ratio $f_{v}=16.9 \%, l=3 \mathrm{~mm}$, $p=4 \mathrm{~mm}, d_{x}=d_{y}=1.22 \mathrm{~mm}, d_{z}=140 \mu \mathrm{m}, R_{x}=R_{z}=$ $380 \mu \mathrm{m},\left(\epsilon_{r f}, \sigma_{f}\right)=\left(5,10^{3} \mathrm{~S} / \mathrm{m}\right),\left(\epsilon_{r m}, \sigma_{m}\right)=(5,0 \mathrm{~S} / \mathrm{m})$.

The relevance of this analogy is verified by computing the shielding effectiveness for both materials using FEM calculations. The calculation conditions are similar to those in Fig. 3 with periodic boundary conditions, except that the composite sheet is replaced by the unit cell of the simple material. The results show good agreement between the shielding properties of the two materials, notably when comparing woven fibers with contact to the two layered laminate composite (see Fig. 10). The variation patterns are equally conserved. Thus, the applicability of analytical EM homogenization rules can be studied by reducing the woven structure to that of a two layered laminate with infinitely long cylindrical inclusions.

\section{AnAlytical homogenization METHODS}

Since the woven fabric material problem can be reduced to that of a two layered composite containing cylindrical inclusions, the corresponding homogenization problem can be solved by using analytical mixing rules.

\section{A. Inclusion-based problems}

In order to choose appropriate formulas for computing the effective properties, the homogenization problem is solved using an inclusion-based method [27], [28] as represented in Fig. 11.

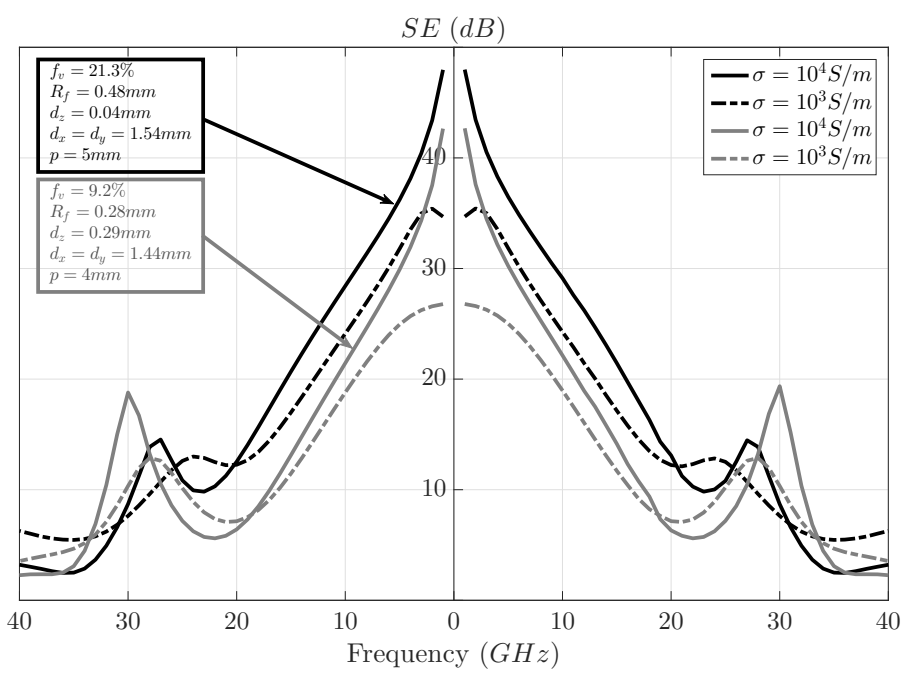

(a) Shielding effectiveness of different woven fiber composites.

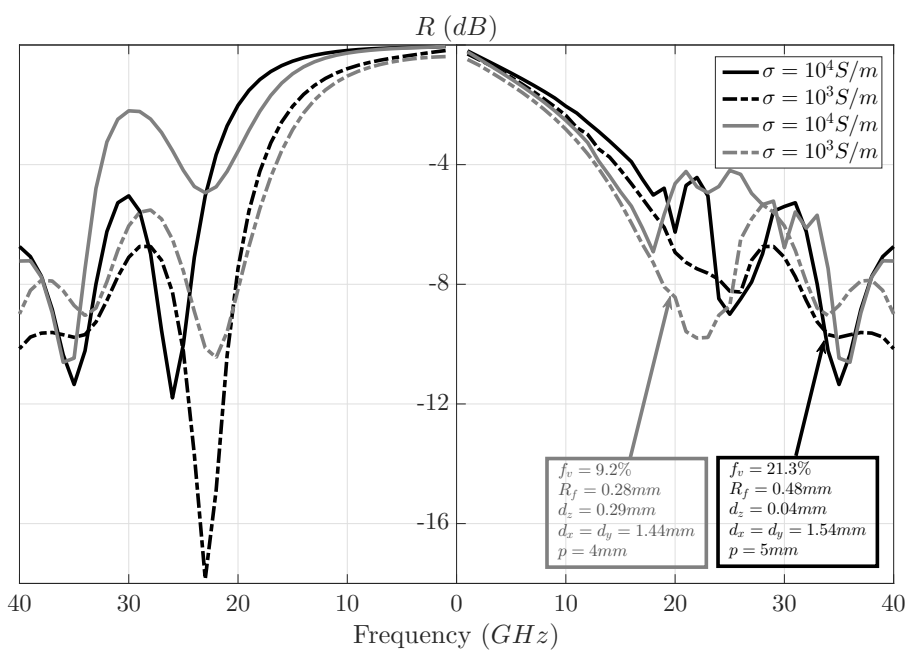

(b) Reflection coefficient of different woven fiber composites.

Fig. 8: FEM (left) and homogenization (right) results for multiple configurations based on Fig. 2. The electric field is polarized in the $\boldsymbol{x}$ direction.
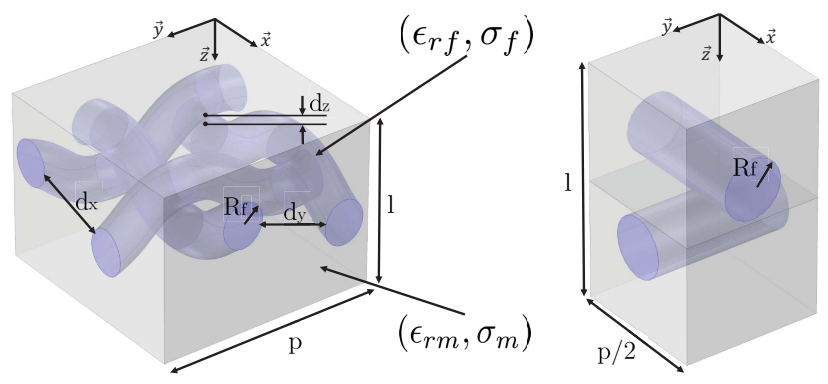

Fig. 9: Woven fiber composite vs. 2 layered unidirectional fiber laminate composite.

Each ellipsoidal phase of the heterogeneous material is located in an unbounded region called infinite medium, which is defined by its electrical properties $\left(\underline{\underline{\epsilon}}_{\infty}^{*}=\underline{\underline{\epsilon}}_{\infty}-\jmath \frac{\underline{\underline{\underline{\sigma}}}}{\omega}\right)$ for 


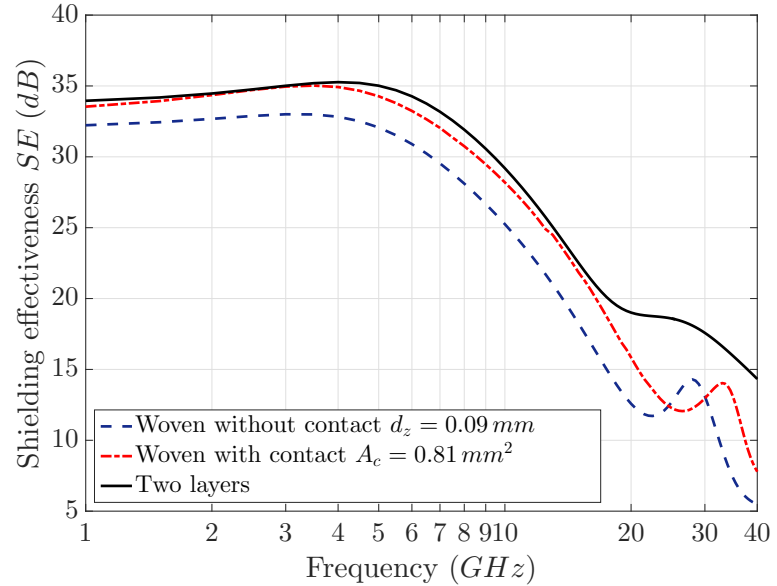

Fig. 10: Shielding effectiveness of an infinite sheet (woven fiber composites vs. two layered composite of Fig. 9) with respect to frequency. Configuration: electric field polarized in the $\boldsymbol{x}$ direction, fiber concentration ratio $f_{v}=16.9 \%, l=$ $3 \mathrm{~mm}, p=4 \mathrm{~mm}, d_{x}=d_{y}=1.22 \mathrm{~mm}, d_{z}=140 \mu \mathrm{m}, R_{f}=$ $380 \mu m,\left(\epsilon_{r f}, \sigma_{f}\right)=\left(5,10^{3} \mathrm{~S} / \mathrm{m}\right),\left(\epsilon_{r m}, \sigma_{m}\right)=(5,0 \mathrm{~S} / \mathrm{m})$.

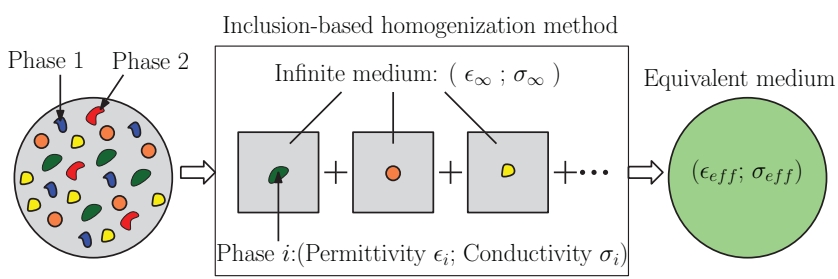

Fig. 11: Inclusion-based homogenization method.

a harmonic excitation. The interaction between the external electric field and the phase is then calculated by introducing the depolarization tensor of the phase $\underline{\underline{N}}_{i}$ as well as its electrical permittivity $\underline{\epsilon}_{i}^{*}$. The average of these individual responses gives the effective permittivity $\underline{\epsilon}_{e f f}^{*}$ [27]

$$
\begin{aligned}
\underline{\underline{\epsilon}}_{e f f}^{*}= & \left\langle\underline{\underline{\epsilon}} i \cdot\left(\underline{\underline{I}}+\underline{\underline{N}}_{i} \cdot \underline{\epsilon}_{\infty}^{*-1} \cdot\left(\underline{\underline{\epsilon}}_{i}^{*}-\underline{\underline{\epsilon}}_{\infty}^{*}\right)\right)^{-1}\right\rangle . \\
& \left\langle\left(\underline{\underline{I}}+\underline{\underline{N_{i}}} \underline{\underline{\underline{\epsilon}}}_{\infty}^{*-1} \cdot\left(\underline{\underline{\epsilon}}_{i}^{*}-\underline{\underline{\epsilon}}_{\infty}^{*}\right)\right)^{-1}\right\rangle
\end{aligned}
$$

where the operator $\langle$.$\rangle denotes a volumetric average operation$ and $\underline{\underline{I}}$ is the second-order identity tensor.

\section{B. Analytical homogenization of two layer composites}

When applying (9) to the laminate composite, the homogenization is divided into three steps as shown in Fig. 12:

a- Layer of fibers parallel to the incident electric field: in this case, the depolarization tensor $\underline{\underline{N_{a}}}$ is written as :

$$
\underline{\underline{N_{a}}}=\left(\begin{array}{ccc}
0 & 0 & 0 \\
0 & \frac{1}{2} & 0 \\
0 & 0 & \frac{1}{2}
\end{array}\right)_{\left(\boldsymbol{u}_{\boldsymbol{x}}, \boldsymbol{u}_{\boldsymbol{y}}, \boldsymbol{u}_{\boldsymbol{z}}\right)}
$$

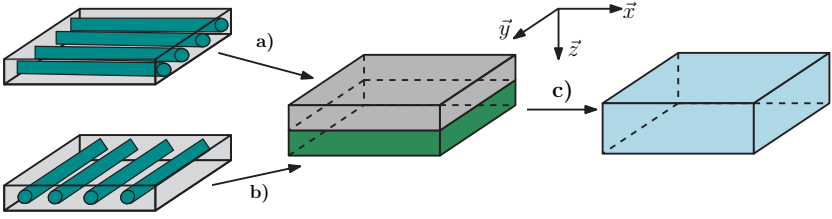

Fig. 12: Homogenization process of a two layered composite.

The effective properties can thus be obtained using the upper bound of Wiener's formula [29].

b- Layer of fibers perpendicular to the incident electric field: the depolarization tensor $\underline{\underline{N_{b}}}$ is expressed as:

$$
\underline{\underline{N_{b}}}=\left(\begin{array}{ccc}
\frac{1}{2} & 0 & 0 \\
0 & 0 & 0 \\
0 & 0 & \frac{1}{2}
\end{array}\right)_{\left(\boldsymbol{u}_{\boldsymbol{x}}, \boldsymbol{u}_{\boldsymbol{y}}, \boldsymbol{u}_{\boldsymbol{z}}\right)}
$$

In this case, to take into account the interaction between the inclusions and incident wave at relatively low volume fractions the infinite medium is chosen as follows [6], [30]:

$$
\epsilon_{\infty}^{*}=\left(\left(\epsilon_{m}+\jmath \frac{\sigma_{m}}{\omega}\right)+\left(\epsilon_{f}+\jmath \frac{\sigma_{f}}{\omega}\right) \cdot\left(\frac{2 R_{f}}{\lambda}\right)^{2}\right) \cdot \underline{\underline{I}}
$$

with $\left(\epsilon_{m}, \sigma_{m}\right)$ and $\left(\epsilon_{f}, \sigma_{f}\right)$ the permittivities of the dielectric matrix and the conductive fibers respectively. $\lambda$ is the wavelength of the incident wave. The effective property in the direction $u$ is then reduced to that of the dynamic homogenization model (DHM) [30]:

$$
\epsilon_{b}^{u}=\frac{\epsilon_{f}^{*} \frac{f_{v}}{\epsilon_{\infty}^{*}+N_{b}^{u}\left(\epsilon_{f}^{*}-\epsilon_{\infty}^{*}\right)}+\epsilon_{m}^{*} \frac{\left(1-f_{v}\right)}{\epsilon_{\infty}^{*}+N_{b}^{u}\left(\epsilon_{m}^{*}-\epsilon_{\infty}^{*}\right)}}{\frac{f_{v}}{\epsilon_{\infty}^{*}+N_{b}^{u}\left(\epsilon_{f}^{*}-\epsilon_{\infty}^{*}\right)}+\frac{\left(1-f_{v}\right)}{\epsilon_{\infty}^{*}+N_{b}^{u}\left(\epsilon_{m}^{*}-\epsilon_{\infty}^{*}\right)}}
$$

c- Two consecutive layers of homogeneous sheets: the depolarization tensor is:

$$
\underline{\underline{N_{c}}}=\left(\begin{array}{lll}
0 & 0 & 0 \\
0 & 0 & 0 \\
0 & 0 & 1
\end{array}\right)_{\left(\boldsymbol{u}_{\boldsymbol{x}}, \boldsymbol{u}_{\boldsymbol{y}}, \boldsymbol{u}_{\boldsymbol{z}}\right)}
$$

The mixing rule for the final step also reduces to Wiener's upper bound formula.

The shielding effectiveness of the equivalent medium is obtained using the formula detailed in equations (2)-(6) and compared to that simulated using FEM (Curves labeled 'Homogenization' and 'FEM:1 fiber' of Fig. 13). The estimate provided by the analytical homogenization is close to that of the simulated heterogeneous material for the lower frequencies. However, as the frequency increases, results start to diverge. The analytical estimates are no longer able to accurately predict the response of the composite.

\section{Representative volume element (RVE) and dynamic effects}

The discrepancies between the numerical and analytical results can be explained. Indeed, analytical mixing rules were established under certain specific assumptions concerning the material and EM solicitation. One of these hypotheses is the existence of a representative volume element [31] which contains a large number of micro heterogeneities. This condition 

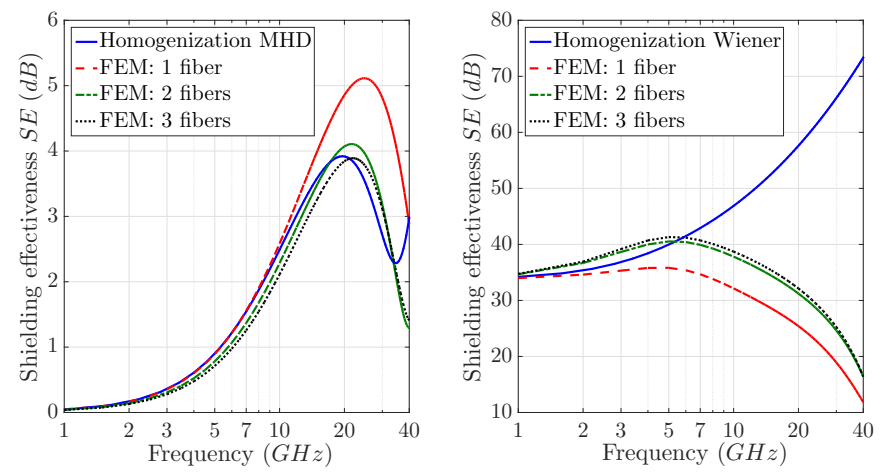

Fig. 13: Evolution of the shielding effectiveness as a function of the number of stacked fibers in the $z$ direction: fibers perpendicular (left) and parallel (right) to the incident electric field. Configuration: fiber concentration ratio $f_{v}=16.9 \%$, $l=3 \mathrm{~mm}, p=4 \mathrm{~mm},\left(\epsilon_{r f}, \sigma_{f}\right)=\left(5,10^{3} \mathrm{~S} / \mathrm{m}\right),\left(\epsilon_{r m}, \sigma_{m}\right)$ $=(5,0 \mathrm{~S} / \mathrm{m})$.

is not satisfied along the $z$ dimension where only two fibers can be found.

To verify the validity of this assumption, each of the two layers of the composite is studied separately. One made of fibers perpendicular to the electric field, and the other containing fibers that are parallel to the electric field. For each laminate, the study consists in consecutively stacking additional layers of inclusion as shown in Fig. 14. At each step the fibers volume fraction remains unchanged (this is done by varying the radius, all other dimensions remaining the same). The fibers occupy a total volume that is proportional to the square of their radius making it not possible to add a fourth layer given the sheet dimensions.

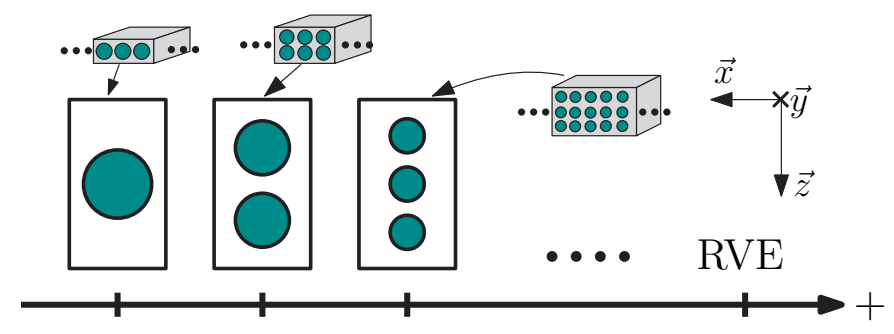

Fig. 14: Stacking fibers in the $z$ direction of the composite in order to form a representative volume element.

The simulated results (Fig. 13) show the evolution of the material response when more fibers are stacked. In fact, the added layers bring the composite closer to satisfying the RVE condition, thus getting the response to converge towards that predicted by the analytical formulas. As for the one layer configuration, it is particularly influenced by the phenomena that occur at higher frequencies, e.g. the proximity and skin effect, which can have a paramount importance on its EM response. That is to say, assuming a quasi-static excitation and the presence of a RVE, mixing rules will predict a shielding effectiveness increase with frequency. However, as frequency increases (and keeping the RVE condition), it is shown in [6] that the skin effect will decrease the shielding effectiveness. Moreover, if now we consider only one layer of fiber in the material, the total induced current will drop even more leading to a decreasing shielding effectiveness with respect to frequency. This explains the trends represented in the figure. This also implies that classical mixing rules cannot currently accurately predict the effective properties of a two layer composite, which means that they are equally not suitable for woven fiber composites. In summary, analytical mixing rules derived from the general homogenization method described by inclusion-based problems is not applicable in this case. This is because the material does not satisfy the RVE condition. Though the homogenization technique carried out in this paper can be computationally heavy, it has shown to give an accurate description of the woven fabric's EM behavior.

\section{CONCLUSION}

In this paper, the electromagnetic response of conductive woven fiber composite materials is studied for applications related to electromagnetic compatibility. For a plane wave configuration, FEM computations demonstrate stable levels of shielding effectiveness for the lower frequency range. Beyond a certain cutoff frequency, results show a drop in the estimated effectiveness. Large scale modeling of composite materials is rendered feasible using electromagnetic homogenization. To this end, a homogenization technique based on finite element simulations on a periodic cell of the microstructure combined with numerical optimization is carried out. Effective electrical properties thereby obtained show accurate description of the woven fiber composites behavior with a mean error margin lower than $12 \%$ over the studied frequency band. Analytical mixing rules are then proved inadequate for estimating an EM effective medium for woven fabric composites at higher frequencies. This is caused by the structure of the composite which, at the mesoscopic scale, contains one layer of fibers. This is not sufficient for the application of analytical mixing rules because they require a representative volume element. While the case of normal incidence is interesting to globally describe shielding levels, an oblique incidence study, which remains to be carried out, would provide a more realistic base for shielding enclosure applications.

\section{ACKNOWLEDGMENT}

This work is supported by the Automotive Mechatronics Chair, a cooperation between Faurecia, CentraleSupélec and Esigelec.

\section{REFERENCES}

[1] A. Sihvola, Electromagnetic Mixing Formulas and Applications, ser. Electromagnetics and Radar Series. Institution of Electrical Engineers, 1999. [Online]. Available: https://books.google.fr/books?id=uIHSNwxBxjgC

[2] K. Bal and V. Kothari, "Permittivity of woven fabrics: A comparison of dielectric formulas for air-fiber mixture," IEEE Transactions on Dielectrics and Electrical Insulation, vol. 17, no. 3, pp. 881-889, Jun. 2010.

[3] C. Holloway, M. Sarto, and M. Johansson, "Analyzing carbon-fiber composite materials with equivalent-layer models," IEEE Transactions on Electromagnetic Compatibility, vol. 47, no. 4, pp. 833-844, nov 2005. 
[4] A. Balzano, I. M. D. Rosa, F. Sarasini, and M. S. Sarto, "Effective properties of carbon fiber composites: EM modeling versus experimental testing," in 2007 IEEE International Symposium on Electromagnetic Compatibility, July 2007, pp. 1-6.

[5] M. Koledintseva, P. C. Rawa, R. Dubroff, J. Drewniak, K. Rozanov, and B. Archambeault, "Engineering of composite media for shields at microwave frequencies," in 2005 International Symposium on Electromagnetic Compatibility, 2005. EMC 2005., vol. 1, Aug 2005, pp. 169174 Vol. 1.

[6] V. Préault, R. Corcolle, L. Daniel, and L. Pichon, "Influence of skin effect on the effective shielding effectiveness of composite materials," Journal of Applied Physics, vol. 115, no. 15, p. 154904, 2014. [Online]. Available: https://doi.org/10.1063/1.4871197

[7] M. H. Nisanci, F. de Paulis, M. Y. Koledintseva, J. L. Drewniak, and A. Orlandi, "From maxwell garnett to debye model for electromagnetic simulation of composite dielectrics - part ii: Random cylindrical inclusions," IEEE Transactions on Electromagnetic Compatibility, vol. 54, no. 2, pp. 280-289, April 2012.

[8] L. Jylha and A. H. Sihvola, "Numerical modeling of disordered mixture using pseudorandom simulations," IEEE Transactions on Geoscience and Remote Sensing, vol. 43, no. 1, pp. 59-64, Jan 2005.

[9] X. Lu, D. Giovanis, J. Yvonnet, V. Papadopoulos, F. Detrez, and J. Bai, "A data-driven computational homogenization method based on neural networks for the nonlinear anisotropic electrical response of graphene/polymer nanocomposites," Computational Mechanics, 10 2018.

[10] M. E. Feddi, Z. Ren, A. Razek, and A. Bossavit, "Homogenization technique for maxwell equations in periodic structures," IEEE Transactions on Magnetics, vol. 33, no. 2, pp. 1382-1385, mar 1997.

[11] M. S. Mirotznik, S. Yarlagadda, R. McCauley, and P. Pa, "Broadband electromagnetic modeling of woven fabric composites," IEEE Transactions on Microwave Theory and Techniques, vol. 60, no. 1, pp. 158-169, Jan 2012.

[12] C. Li, D. Lesselier, and Y. Zhong, "Scattering of obliquely incident electromagnetic plane waves by composite panel involving periodic arrays of circular fibers," IEEE Transactions on Antennas and Propagation, vol. 63, no. 7, pp. 3168-3178, July 2015.

[13] R. Sutthaweekul, A. M. J. Marindra, and G. Y. Tian, "Study of microwave responses on layered woven cfrp composites," in 2017 9th International Conference on Information Technology and Electrical Engineering (ICITEE), Oct 2017, pp. 1-5.

[14] L. Diaz Angulo, M. Ruiz Cabello, J. Alvarez, A. R. Bretones, and S. G. García, "From microscopic to macroscopic description of composite thin panels: A road map for their simulation in time domain," IEEE Transactions on Microwave Theory and Techniques, vol. 66, no. 2, pp. 660-668, Feb 2018.

[15] F. D. Senghor, G. Wasselynck, H. K. Bui, S. Branchu, D. Trichet, and G. Berthiau, "Electrical conductivity tensor modeling of stratified woven-fabric carbon fiber reinforced polymer composite materials," IEEE Transactions on Magnetics, vol. 53, no. 6, pp. 1-4, June 2017.

[16] M. S. Sarto, S. Greco, and A. Tamburrano, "Shielding effectiveness of protective metallic wire meshes: Em modeling and validation," IEEE Transactions on Electromagnetic Compatibility, vol. 56, no. 3, pp. 615621, June 2014.

[17] S. Greco, A. Tamburrano, A. D'Aloia, R. Mufatti, and M. S. Sarto, "Shielding effectiveness properties of carbon-fiber reinforced composite for hirf applications," in International Symposium on Electromagnetic Compatibility - EMC EUROPE, Sep. 2012, pp. 1-6.

[18] M. J. Akhtar, L. E. Feher, and M. Thumm, "A waveguide-based twostep approach for measuring complex permittivity tensor of uniaxial composite materials," IEEE Transactions on Microwave Theory and Techniques, vol. 54, no. 5, pp. 2011-2022, May 2006.

[19] G. Al Achkar, L. Pichon, N. Benjelloun, and L. Daniel, "A broadband electromagnetic homogenization method for composite materials," IEEE Transactions on Magnetics, vol. 54, no. 3, pp. 1-4, March 2018.

[20] S. Celozzi, R. Araneo, and G. Lovat, Electromagnetic Shielding. John Wiley \& Sons, Ltd, 2008, pp. i-xii. [Online]. Available: https://onlinelibrary.wiley.com/doi/abs/10.1002/9780470268483.fmatter

[21] J. Stratton, I. Antennas, and P. Society, Electromagnetic Theory, ser. An IEEE Press classic reissue. Wiley, 2007. [Online]. Available: https://books.google.fr/books?id=zFeWdS2luE4C

[22] Z. Michalewicz, Genetic Algorithms + Data Structures = Evolution Programs, revised and extended edition, Eds. 3rd. edition, New York : Springer, 1996.

[23] J.-P. Berenger, "A perfectly matched layer for the absorption of electromagnetic waves," Journal of Computational Physics, vol. 114, no. 2, pp. 185 - 200, 1994. [Online]. Available: http://www.sciencedirect.com/science/article/pii/S0021999184711594

[24] H. Hertz, "J. reine angew. math. 92, 156. reprinted in an english translation in hertz's' miscellaneous papers'," 1881.

[25] R. Holm, Electric contacts: theory and application. Springer Science \& Business Media, 2013.

[26] C. C. Roussos and J. Swingler, "A 3d contact analysis approach for the visualization of the electrical contact asperities," AIP Advances, vol. 7, no. 1, p. 015023, 2017. [Online]. Available: https://doi.org/10.1063/1.4974151

[27] A. Sihvola and I. Lindell, "Electrostatics of an anisotropic ellipsoid in an anisotropic environment," AE International Journal of Electronics and Communications, vol. 50, pp. 289-292, 091996.

[28] R. Corcolle, L. Daniel, and F. Bouillault, "Generic formalism for homogenization of coupled behavior: Application to magnetoelectroelastic behavior," Phys. Rev. B, vol. 78, p. 214110, Dec 2008. [Online]. Available: https://link.aps.org/doi/10.1103/PhysRevB.78.214110

[29] L. Daniel and R. Corcolle, "A note on the effective magnetic permeability of polycrystals," IEEE Transactions on Magnetics, vol. 43, no. 7, pp. 3153-3158, July 2007.

[30] V. Préault, R. Corcolle, L. Daniel, and L. Pichon, "Effective permittivity of shielding composite materials for microwave frequencies," IEEE Transactions on Electromagnetic Compatibility, vol. 55, no. 6, pp. 11781186, Dec 2013.

[31] T. Kanit, S. Forest, I. Galliet, V. Mounoury, and D. Jeulin, "Determination of the size of the representative volume element for random composites: statistical and numerical approach," International Journal of Solids and Structures, vol. 40, no. 13, pp. 3647 - 3679, 2003. [Online]. Available: http://www.sciencedirect.com/science/article/pii/S0020768303001434 